\title{
Research Article \\ On the Complex Zeros of Some Families of Orthogonal Polynomials
}

\section{Eugenia N. Petropoulou}

Division of Applied Mathematics and Mechanics, Department of Engineering Sciences, University of Patras, 26500 Patras, Greece

Correspondence should be addressed to Eugenia N. Petropoulou, jenpetro@des.upatras.gr

Received 8 March 2010; Accepted 6 May 2010

Academic Editor: Roman Šimon Hilscher

Copyright (C) 2010 Eugenia N. Petropoulou. This is an open access article distributed under the Creative Commons Attribution License, which permits unrestricted use, distribution, and reproduction in any medium, provided the original work is properly cited.

The complex zeros of the orthogonal Laguerre polynomials $L_{n}^{(a)}(x)$ for $a<-n$, ultraspherical polynomials $P_{n}^{(\lambda)}(x)$ for $\lambda<-n$, Jacobi polynomials $P_{n}^{(a, \beta)}(x)$ for $a<-n, \beta<-n, a+\beta<-2(n+1)$, orthonormal Al-Salam-Carlitz II polynomials $P_{n}^{(a)}(x ; q)$ for $a<0,0<q<1$, and $q$-Laguerre polynomials $L_{n}^{(a)}(x ; q)$ for $a<-n, 0<q<1$ are studied. Several inequalities regarding the real and imaginary properties of these zeros are given, which help locating their position. Moreover, a few limit relations regarding the asymptotic behavior of these zeros are proved. The method used is a functional analytic one. The obtained results complement and improve previously known results.

\section{Introduction}

Orthogonal polynomials appear naturally in various problems of physics and mathematics and are considered as one of the basic tools in confronting problems of mathematical physics. Also, orthogonal polynomials have many important applications in problems of numerical analysis, such as interpolation or optimization. For a survey on applications and computational aspects of orthogonal polynomials, see [1] and the references therein. following.

Some of the most important properties of orthogonal polynomials, $P_{n}(x)$, are the

(P1) The orthogonal polynomials $P_{n}(x)$ are orthogonal with respect to a weight function $\rho(x)>0$ on an interval of orthogonality $[a, b] \subseteq \mathbb{R}$ and all their zeros are real and simple and lie inside $(a, b)$.

(P2) Some classes of orthogonal polynomials $P_{n}(x)$ (including some of the classes studied in the present paper) satisfy an ordinary differential equation of the form

$$
\sigma(x) P_{n}^{\prime \prime}(x)+\tau(x) P_{n}^{\prime}(x)+\lambda_{n} P_{n}(x)=0,
$$


where $\sigma(x)$ is a polynomial of degree at most two, $\tau(x)$ is a polynomial of degree exactly one, and $\lambda_{n}$ is a constant.

(P3) The orthogonal polynomials $P_{n}(x)$ satisfy a three-term recurrence relation of the form

$$
\begin{gathered}
\tilde{\alpha}_{n} P_{n+1}(x)+\tilde{\beta}_{n} P_{n}(x)+\tilde{c}_{n} P_{n-1}(x)=x P_{n}(x), \quad n=0,1,2, \ldots, \\
P_{-1}(x)=0, \quad P_{0}(x)=1,
\end{gathered}
$$

where $\tilde{\alpha}_{n-1} \tilde{\gamma}_{n}>0$.

An analog to the theory of classical orthogonal polynomials has recently been developed for $q$-polynomials, $0<q<1$, which also appear in various areas of mathematics and physics. The $q$-polynomials satisfy also a recurrence relation of the form (1.2), but now the sequences $\tilde{a}_{n}, \widetilde{\beta}_{n}$ and $\widetilde{c}_{n}$ as well as the polynomials $P_{n}(x)$ depend on the parameter $q, 0<q<1$. On the other hand the $q$-polynomials do not satisfy a differential equation, but a $q$-difference equation which is considered as the $q$-analog of (1.1). For more information on classical or $q$-polynomials one may consult [2-6] and the references therein. Also, q-polynomials arise in the context of indeterminate moment problems. In this case, there are some classes of orthogonal polynomials for which the corresponding measure of orthogonality is not unique. This may give rise to various types of $q$-polynomials, other than the ones studied in the present paper. For more information see $[4,7-9]$ and the references therein.

Due to their importance, orthogonal polynomials have drawn the attention of many researchers and there is a renewed interest for them and their properties during the last 2030 years. Many of the results regarding orthogonal polynomials, concern the properties of their zeros, such as their monotonicity, concavity, or convexity. This interest in the zeros of the orthogonal polynomials stimulates due to the physical interpretation of their zeros, such as their electrostatic interpretation and their appearance in various physical problems; see [4] and the references therein.

There are several ways to deal with problems involving the properties of the zeros of $P_{n}(x)$. Among them are methods

(M1) of real analysis utilizing the formulae of $P_{n}(x)$ and their properties,

(M2) which utilize the differential equation (1.1) when $x \in \mathbb{R}$,

(M3) which utilize the recurrence relation (1.2) when $\tilde{\alpha}_{n}, \widetilde{\beta}_{n}$, and $\tilde{\gamma}_{n}$ are real sequences,

(M4) of functional analysis which transform the problem of the zeros of $P_{n}(x)$ to the equivalent problem of the eigenvalues of a specific linear operator by using (1.2), regardless of the type (complex or real) of the sequences $\widetilde{\alpha}_{n}, \widetilde{\beta}_{n}$, and $\tilde{\gamma}_{n}$.

In most cases, the orthogonal polynomials depend on at least one parameter which appears in the formulae of $\sigma(x)$ and/or $\tau(x)$ in (1.1), or in the formulae of $\widetilde{\alpha}_{n}$ and /or $\tilde{\beta}_{n}$ and/or $\tilde{\gamma}_{n}$ in (1.2), or in the formula of the weight function $\rho(x)$ and which influence the behavior of the zeros of $P_{n}(x)$. In order $\rho(x)$ to be positive, or $\widetilde{\alpha}_{n-1} \tilde{\gamma}_{n}>0$ or even more $\widetilde{\alpha}_{n}, \tilde{\beta}_{n}$, and $\tilde{\gamma}_{n}$ to be real, the involved parameters should satisfy specific assumptions (usually simple inequalities). Otherwise, the first property (P1) of the $P_{n}(x)$ may not hold and since $P_{n}(x)$ are defined recursively by (1.2), if $\widetilde{\alpha}_{n}, \tilde{\beta}_{n}$, and $\tilde{\gamma}_{n}$ are complex sequences, the polynomials $P_{n}(x)$ will no longer be real polynomials. In this case, their zeros will no longer be exclusively 
real and there is a need to locate their position. Moreover, the usual methods (M1)-(M3) mentioned before for the study of the zeros of $P_{n}(x)$ may not apply at all, when $P_{n}(x)$ are complex, or they may need serious modifications. Instead, the (M4) method can be used directly.

Such a functional analytic method was introduced in [10] and was successfully used in a series of papers by the authors of [10] and their collaborators, including paper [11], where results were given regarding the real part of the complex zeros of a class of polynomials including the generalized Bessel polynomials. The most recent application of this method was in $[12,13]$, where convexity results and differential inequalities were deduced for the largest and lowest zeros and functions involving these zeros of several $q$-polynomials. This method is also used in the present paper and it is briefly presented in Section 3 . The main idea is to transform the problem of the zeros of $P_{n}(x)$ satisfying (1.2) to the equivalent problem of the eigenvalues of a specific tridiagonal operator $T$. Then, by utilizing the properties of $T$, several properties of the zeros of $P_{n}(x)$ can be proved.

The aim of the present paper is to provide regions (in $\mathbb{C}$ ) of the location of the complex zeros of the following:

(i) Laguerre orthogonal polynomials $L_{n}^{(a)}(x)$ for $a<-n$,

(ii) ultraspherical orthogonal polynomials $P_{n}^{(\lambda)}(x)$ for $\lambda<-n$,

(iii) Jacobi orthogonal polynomials $P_{n}^{(a, \beta)}(x)$ for $a<-n, \beta<-n, a+\beta<-2(n+1)$,

(iv) orthonormal Al-Salam-Carlitz II polynomials $P_{n}^{(a)}(x ; q)$ for $a<0,0<q<1$,

(v) $q$-Laguerre orthonormal polynomials $L_{n}^{(a)}(x ; q)$ for $a<-n, 0<q<1$.

These regions are given in the form of inequalities regarding the real and imaginary properties of the zeros of the polynomials under consideration. Moreover, a few limit relations regarding the asymptotic behavior of these zeros are given. All these results are stated in Section 2 and proved in Section 4. The reason for choosing the above mentioned five classes of orthogonal polynomials, apart from pure mathematical curiosity, is the fact that their zeros and especially the zeros of the Jacobi and Laguerre polynomials admit a very interesting electrostatic interpretation (see, e.g., [14-16], [6, page 140] and the references therein).

To the best of the author's knowledge there are very few results concerning the location of the complex zeros of the classical or $q$-polynomials or their limit relations. More precisely, in the thesis [17] and the paper [18], the behavior of the complex zeros of the Laguerre, $q$-Laguerre, and Jacobi polynomials is primarily studied. Among others, an inequality regarding the real part of the zeros of the Laguerre polynomials and limit relations regarding the zeros of the Laguerre, $q$-Laguerre and Jacobi polynomials are proved using their explicit formulae and their recurrence relations. Also in [19], the zeros of the hypergeometric polynomial $F(-n, b ; 2 b ; z)$, for $b>-1 / 2$ are studied. These results are then applied in order to obtain information for the zeros of the Ultraspherical (for $\lambda<-n$ ) and Jacobi (for $\beta=-(1 / 2)(a+1)-n, a>-2$ and for $a=-2 \beta-2 n-1, \beta>-1$ ) polynomials. Finally in [20], the zeros of the Ultraspherical polynomials are further investigated. More precisely, the authors give a description of the trajectories of the zeros as $\lambda$ decreases from $-1 / 2$ to $1-n$. Several useful figures created using Mathematica illustrate these trajectories when $n=8$. In the end, the authors conclude that "as $\lambda$ descends below -7 , all 8 zeros of $P_{8}^{\lambda}(x)$ are on the imaginary axis tending symmetrically to the origin as $\lambda \rightarrow-\infty$ ". 
The results of the present paper (specifically Theorems 2.1 and 2.4) complement and improve the results of $[17,19,20]$.

\section{Main Results}

In this section, several theorems are stated regarding the complex zeros of the orthogonal Laguerre, Ultraspherical and Jacobi, as well as the orthonormal Al-Salam-Carlitz II and $q$ Laguerre polynomials. In each case, a region of the complex plane is given where these zeros lie, as well as a few limit relations regarding their asymptotic behavior. The proofs of these theorems are given in Section 4.

Theorem 2.1. The zeros $x_{n k}(a)=\operatorname{Re}\left[x_{n k}(a)\right]+i \operatorname{Im}\left[x_{n k}(a)\right]$ of the Laguerre orthogonal polynomials $L_{n}^{(a)}(x)$ for $a<-n$ satisfy the following relations:

$$
\begin{gathered}
a+1 \leq \operatorname{Re}\left[x_{n k}(a)\right] \leq 2 n+a-1, \\
\left|\operatorname{Im}\left[x_{n k}(a)\right]\right| \leq 2 \sqrt{-n(a+1)}
\end{gathered}
$$

Moreover,

$$
\lim _{a \rightarrow-\infty} \frac{x_{n k}(a)}{a}=1
$$

Remark 2.2. It is obvious from (2.1) that if $a<1-2 n$, then $\operatorname{Re}\left[x_{n k}(a)\right] \leq 0$.

Remark 2.3. In [17, pages 112-131], using the explicit formula for the Laguerre polynomials and their recurrence relation, the inequality (2.1) was obtained, among other interesting relations. Moreover it was proved that

$$
\lim _{a \rightarrow-\infty}\left|\frac{x_{n k}(a)}{a}\right|=1
$$

Notice that relation (2.3) is stronger than (2.4).

Theorem 2.4. The zeros $x_{n k}(\lambda)$ of the Ultraspherical orthogonal polynomials $P_{n}^{(\lambda)}(x)$ for $\lambda<-n$ are purely imaginary. Moreover

$$
\begin{gathered}
\left|x_{n k}(\lambda)\right| \leq \sqrt{\frac{-2 \lambda n}{(n+\lambda)(\lambda+n-1)}}, \\
\lim _{\lambda \rightarrow-\infty} x_{n k}(\lambda)=0 .
\end{gathered}
$$

Remark 2.5. In [19], as a consequence of a more general result regarding the zeros of the hypergeometric function $F(-n, b ; 2 b ; z)$, it was proved that all zeros of the Ultraspherical polynomials are purely imaginary for $\lambda<1-n$, which is slightly stronger than the inequality $\lambda<-n$. In [20], the zeros of the Ultraspherical polynomials are further investigated. More 
precisely, the authors give a description of the trajectories of the zeros as $\lambda$ decreases from $-1 / 2$ to $1-n$. Several useful figures created using Mathematica illustrate these trajectories when $n=8$. In the end the authors conclude that "as $\lambda$ descends below -7 , all 8 zeros of $P_{8}^{\lambda}(x)$ are on the imaginary axis tending symmetrically to the origin as $\lambda \rightarrow-\infty$ ", which is in accordance with the more general result (2.6).

Theorem 2.6. The zeros $x_{n k}(a, \beta)=\operatorname{Re}\left[x_{n k}(a, \beta)\right]+i \operatorname{Im}\left[x_{n k}(a, \beta)\right]$ of the Jacobi orthogonal polynomials $P_{n}^{(a, \beta)}(x)$ for $a<-n, \beta<-n, a+\beta<-2(n+1)$ satisfy the following relations:

$$
\begin{gathered}
\frac{\beta^{2}-a^{2}}{(2 n+a+\beta)(2 n+a+\beta-2)} \leq \operatorname{Re}\left[x_{n k}(a, \beta)\right] \leq \frac{\beta-a}{a+\beta+2}, \quad \text { for } \beta>a, \\
\frac{\beta-a}{a+\beta+2} \leq \operatorname{Re}\left[x_{n k}(a, \beta)\right] \leq \frac{\beta^{2}-a^{2}}{(2 n+a+\beta)(2 n+a+\beta-2)}, \quad \text { for } \beta<a, \\
\left|\operatorname{Im}\left[x_{n k}(a, \beta)\right]\right| \leq 2 \sqrt{\frac{-4(a+1)(\beta+1)(a+\beta+1) n}{\left[(2 n+a+\beta)^{2}-1\right](2 n+a+\beta)^{2}}} .
\end{gathered}
$$

Moreover,

$$
\begin{gathered}
\lim _{a \rightarrow-\infty} x_{n k}(a, \beta)=-1, \quad \lim _{\beta \rightarrow-\infty} x_{n k}(a, \beta)=1, \\
\lim _{a \rightarrow-\infty} \frac{x_{n k}(a, \beta)}{a}=0=\lim _{\beta \rightarrow-\infty} \frac{x_{n k}(a, \beta)}{\beta} .
\end{gathered}
$$

Remark 2.7. It is obvious from (2.7) that the sign of $\operatorname{Re}\left[x_{n k}(a, \beta)\right]$ depends on the sign of $\beta-a$, that is, $\operatorname{Re}\left[x_{n k}(a, \beta)\right]>0$ if $\beta<a$ and $\operatorname{Re}\left[x_{n k}(a, \beta)\right]<0$ if $\beta>a$.

Remark 2.8. It is well known, see, for example, [4, page 99], that the Laguerre polynomials are a limiting case of the Jacobi polynomials, by first putting the Jacobi weight function on $[0, a]$ and then letting $a \rightarrow \infty$. Thus, it is obvious that Theorem 2.1 cannot be obtained from Theorem 2.6, since that would require taking the limit for $a \rightarrow \infty$, which cannot hold since $a<-n<0$.

Remark 2.9. It is well known, see, for example, [4, page 94] or [5, page 40], that the Ultraspherical polynomials are Jacobi polynomials for $a=\beta=\lambda-(1 / 2)$. By applying Theorem 2.6 for $a=\beta=\lambda-(1 / 2)$ one obtains that "the zeros $x_{n k}(\lambda)$ of the Ultraspherical orthogonal polynomials $P_{n}^{(\lambda)}(x)$ for $\lambda<-n-(1 / 2)$ are purely imaginary and

$$
\left|x_{n k}(\lambda)\right| \leq 2 \sqrt{\frac{-2 \lambda n}{(n+\lambda)(\lambda+n-1)}}\left|\frac{\lambda+1 / 2}{2 \lambda+2 n-1}\right|, \quad \lim _{\lambda \rightarrow-\infty} x_{n k}(\lambda)=0 . "
$$

This result, however, is slightly worse than Theorem 2.4 and this is the reason that the Ultraspherical polynomials are treated in their own and not as a specific case of the Jacobi polynomials. 
Theorem 2.10. The zeros $x_{n k}(a ; q)=\operatorname{Re}\left[x_{n k}(a ; q)\right]+i \operatorname{Im}\left[x_{n k}(a ; q)\right]$ of the orthonormal Al-SalamCarlitz II polynomials $P_{n}^{(a)}(x ; q)$ for $a<0,0<q<1$ satisfy the following relations:

$$
\begin{gathered}
\frac{a+1}{q^{n-1}} \leq \operatorname{Re}\left[x_{n k}(a ; q)\right] \leq a+1, \quad \text { for } a \leq-1, \\
a+1 \leq \operatorname{Re}\left[x_{n k}(a ; q)\right] \leq \frac{a+1}{q^{n-1}}, \text { for }-1<a<0, \\
\left|\operatorname{Im}\left[x_{n k}(a ; q)\right]\right| \leq 2 \sqrt{\frac{(-a)\left(1-q^{n}\right)}{q^{2 n-1}}} .
\end{gathered}
$$

Moreover,

$$
\lim _{a \rightarrow-\infty} \frac{x_{n k}(a ; q)}{a^{2}}=0
$$

Remark 2.11. It is obvious from (2.12) that if $-1<a<0$, then $\operatorname{Re}\left[x_{n k}(a ; q)\right] \geq 0$, whereas if $a \leq-1$, then $\operatorname{Re}\left[x_{n k}(a ; q)\right] \leq 0$.

Theorem 2.12. The zeros $x_{n k}(a ; q)=\operatorname{Re}\left[x_{n k}(a ; q)\right]+i \operatorname{Im}\left[x_{n k}(a ; q)\right]$ of the q-Laguerre orthonormal polynomials $L_{n}^{(a)}(x ; q)$ for $a<-n, 0<q<1$ satisfy the following relations:

$$
\begin{gathered}
\frac{1-q^{a+1}}{q^{2 n+a-1}} \leq \operatorname{Re}\left[x_{n k}(a ; q)\right] \leq \frac{1+q}{q^{2 n+a-1}}-\frac{q^{n+a}+q^{n}}{q^{a+1}} \\
\left|\operatorname{Im}\left[x_{n k}(a ; q)\right]\right| \leq 2 \sqrt{\frac{\left(1-q^{n}\right)\left(q^{a+1}-1\right)}{q^{4 n+2 a-1}}} .
\end{gathered}
$$

\section{The Method}

A sequence $\left\{P_{n}(x)\right\}_{n=0}^{\infty}$ of orthogonal polynomials satisfies a three-term recurrence relation of the following form:

$$
\begin{gathered}
\tilde{\alpha}_{n} P_{n+1}(x)+\tilde{\beta}_{n} P_{n}(x)+\tilde{c}_{n} P_{n-1}(x)=x P_{n}(x), \quad n=0,1,2, \ldots, \\
P_{-1}(x)=0, \quad P_{0}(x)=1 .
\end{gathered}
$$

However, after specific transformations, relation (3.1) can take the following form:

$$
\begin{gathered}
\alpha_{n} Q_{n+1}(x)+\alpha_{n-1} Q_{n-1}(x)+\beta_{n} Q_{n}(x)=x Q_{n}(x), \quad n=1,2, \ldots, \\
Q_{0}(x)=0, \quad Q_{1}(x)=1 .
\end{gathered}
$$

The same holds for $q$-polynomials. The only difference is that now the sequences $\tilde{a}_{n}, \tilde{\beta}_{n}, \tilde{c}_{n}, \alpha_{n}$ and $\beta_{n}$ as well as the polynomials $P_{n}(x)$ and $Q_{n}(x)$ depend also on the parameter $q, 0<q<1$. 
The method used in this paper for the study of the zeros of the polynomials $P_{n}(x)$ is a functional-analytic one, based on the equivalent transformation of the problem of the zeros of $P_{n}(x)$ to the problem of the eigenvalues of a specific linear operator. More precisely, let $\left\{e_{k}\right\}_{k=1}^{n}$ be an orthonormal base in a finite dimensional Hilbert space $H_{n}$ with inner product denoted as usual by $(\cdot, \cdot)$ and let $V$ be the truncated shift operator:

$$
V e_{k}=e_{k+1}, \quad k=1, \ldots, n-1, \quad V e_{n}=0
$$

The adjoint of $V$ is the shift operator $V^{*}$ defined by

$$
V^{*} e_{k}=e_{k-1}, \quad k=2, \ldots, n, \quad V^{*} e_{1}=0 .
$$

Let also $A$ and $B$ be the diagonal operators:

$$
A e_{k}=\alpha_{k} e_{k}, \quad B e_{k}=\beta_{k} e_{k}, \quad k=1, \ldots, n
$$

It is known (see, e.g., [10]) that the zeros of the polynomials $Q_{n+1}(x)$ defined by (3.2) are the eigenvalues of the operator $T=A V^{*}+V A+B$, that is,

$$
T f_{k}=x_{n k} f_{k}, \quad\left\|f_{k}\right\|=1
$$

and vice versa. In the case where the polynomials $Q_{n+1}(x)$ depend on a parameter, the eigenvalues $x_{n k}$ and the corresponding eigenvectors $f_{k}$ depend also on the same parameter.

For technical reasons one may choose, instead, the orthonormal base $\left\{E_{k}\right\}_{k=0}^{n-1}$ of $H_{n}$. In this case the operators $V, V^{*}, A$, and $B$ are defined as follows:

$$
\begin{gathered}
V E_{k}=E_{k+1}, \quad k=0, \ldots, n-2, \quad V E_{n-1}=0, \\
V^{*} E_{k}=E_{k-1}, \quad k=1, \ldots, n-1, \quad V^{*} E_{0}=0, \\
A E_{k}=\alpha_{k} E_{k}, \quad B E_{k}=\beta_{k} E_{k}, \quad k=0, \ldots, n-1,
\end{gathered}
$$

and the zeros of the polynomials $Q_{n}(x)$ are the eigenvalues of the operator $T$. In the rest of the paper it will be obvious from the text which base (and as a consequence which definition of the above mentioned operators) is used.

It worths mentioning at this point that.

(i) If the sequences $\alpha_{n}$ and $\beta_{n}$ are real, then the operator $T=A V^{*}+V A+B$ is a selfadjoint operator and thus its eigenvalues are all real. Moreover, it follows easily from (3.6) that

$$
x_{n k}=\left(T f_{k}, f_{k}\right) .
$$

From (3.8), several useful information and inequalities regarding $x_{n k}$ can be deduced. 
(ii) If $\alpha_{n}$ is purely imaginary and $\beta_{n}$ real, then $T^{*}=V A^{*}+A^{*} V^{*}+B$ and thus $T$ is obviously not a self-adjoint operator. However, by setting $S=(1 / i)\left(A V^{*}+V A\right), T$ takes the form $T=i S+B$, where both $S$ and $B$ are self-adjoint operators. Then it follows easily from (3.6) that

$$
x_{n k}=\left(B f_{k}, f_{k}\right)+i\left(S f_{k}, f_{k}\right) \text {. }
$$

Since $S$ and $B$ are both self-adjoint operators, the inner products $\left(B f_{k}, f_{k}\right)$ and $\left(S f_{k}, f_{k}\right)$ are both real. From (3.9), several useful information and inequalities regarding $x_{n k}$ can be deduced, like those mentioned in Theorems 2.1-2.12. The proofs of these theorems rely on relation (3.9) and inequalities depending on the formulae of $\alpha_{n}$ and $\beta_{n}$.

\section{Proofs}

Proof of Theorem 2.1. The Laguerre polynomials $L_{n}^{(a)}(x)$ satisfy the recurrence relation (3.1) for

$$
\tilde{\alpha}_{n}=-(n+1), \quad \widetilde{\beta}_{n}=a+2 n+1, \quad \tilde{c}_{n}=-(a+n) .
$$

However, by setting first $L_{n}^{a}(x)=R_{n+1}^{(a)}(x)$ and then $R_{n}^{(a)}(x)=(-1)^{n+1} \sqrt{U_{n}} Q_{n}^{(a)}(x)$, where $U_{0}=0, U_{1}=1$, and $U_{n}=((n+a-1) /(n-1)) U_{n-1}$, for $n \geq 2$, one obtains as in [21] a relation for $Q_{n}^{(a)}(x)$ of the form (3.2) with

$$
\alpha_{n}=\sqrt{n(n+a)}, \quad \beta_{n}=2 n+a-1
$$

For $a<-n$, the sequence $\alpha_{n}$ is purely imaginary and can be rewritten as $\alpha_{n}=i \sqrt{n(-n-a)}$. It is obvious from what already mentioned that the zeros $x_{n k}(a)$ of $L_{n}^{(a)}(x)$ are the same with the zeros of $Q_{n+1}^{(a)}(x)$ and, as a consequence, are the same as the eigenvalues of the operator $T=A V^{*}+V A+B$, where

$$
A e_{k}=i \sqrt{k(-k-a)} e_{k}, \quad B e_{k}=(2 k+a-1) e_{k}, \quad k=1, \ldots, n
$$

Due to (3.9), it is obvious that

$$
\operatorname{Re}\left[x_{n k}(a)\right]=\left(B f_{k}, f_{k}\right), \quad \operatorname{Im}\left[x_{n k}(a)\right]=\left(S f_{k}, f_{k}\right) .
$$

Since

$$
\begin{gathered}
\left(B f_{k}, f_{k}\right)=\sum_{l=1}^{n} \beta_{l}\left|\left(f_{k}, e_{l}\right)\right|^{2}, \\
a+1 \leq \beta_{l} \leq 2 n+a-1,
\end{gathered}
$$


it follows that

$$
(a+1)\left\|f_{k}\right\|^{2} \leq\left(B f_{k}, f_{k}\right) \leq(2 n+a-1)\left\|f_{k}\right\|^{2} \Longrightarrow a+1 \leq \operatorname{Re}\left[x_{n k}(a)\right] \leq 2 n+a-1 .
$$

Regarding the imaginary part of $x_{n k}(a)$, it follows using the Schwarz inequality that

$$
\left|\operatorname{Im}\left[x_{n k}(a)\right]\right| \leq\|S\| \leq 2\|A\|=2 \sup _{k} \sqrt{-k(a+k)} \leq 2 \sqrt{-n(a+1)} .
$$

From (2.1) and (2.2) it follows that (since $a<0$ )

$$
\left.\begin{array}{c}
1+\frac{2 n-1}{a} \leq \frac{\operatorname{Re}\left[x_{n k}(a)\right]}{a} \leq 1+\frac{1}{a} \\
2 \frac{\sqrt{-n(a+1)}}{a} \leq \frac{\operatorname{Im}\left[x_{n k}(a)\right]}{a} \leq-2 \frac{\sqrt{-n(a+1)}}{a}
\end{array}\right\} \Longrightarrow \lim _{a \rightarrow-\infty} \frac{x_{n k}(a)}{a}=1 .
$$

Proof of Theorem 2.4. The Ultraspherical polynomials $P_{n}^{(\lambda)}(x)$ satisfy the recurrence relation (3.1) for

$$
\tilde{\alpha}_{n}=\frac{n+1}{2(n+\lambda)}, \quad \tilde{\beta}_{n}=0, \quad \tilde{c}_{n}=\frac{n+2 \lambda-1}{2(n+\lambda)} .
$$

However, by setting first $P_{n}^{\lambda}(x)=R_{n+1}^{(\lambda)}(x)$ and then $R_{n}^{(\lambda)}(x)=\sqrt{n U_{n} /(n+\lambda-1)} Q_{n}^{(\lambda)}(x)$, where $U_{0}=0, U_{1}=\lambda$, and $U_{n}=((n+2 \lambda-2) / n) U_{n-1}$, for $n \geq 2$, one obtains as in [22] a relation for $Q_{n}^{(\lambda)}(x)$ of the form (3.2) with

$$
\alpha_{n}=\frac{1}{2} \sqrt{\frac{n(n+2 \lambda-1)}{(n+\lambda)(n+\lambda-1)}}, \quad \beta_{n}=0 .
$$

For $\lambda<-n$, the sequence $\alpha_{n}$ is purely imaginary and can be rewritten as $\alpha_{n}=$ $i(1 / 2) \sqrt{(-n)(2 \lambda+n-1) /(n+\lambda)(n+\lambda-1)}$. As before the zeros $x_{n k}(\lambda)$ of $P_{n}^{(\lambda)}(x)$ are the same with the eigenvalues of the operator $T=A V^{*}+V A+B$, where

$$
A e_{k}=i \sqrt{\frac{(-k)(2 \lambda+k-1)}{(k+\lambda)(k+\lambda-1)}} e_{k}, \quad B e_{k}=0, \quad k=1, \ldots, n
$$

Due to (3.9), it is obvious that the zeros $x_{n k}(\lambda)$ of $P_{n}^{(\lambda)}(x)$ are purely imaginary and that

$$
x_{n k}(\lambda)=i\left(S f_{k}, f_{k}\right)
$$


As before, it follows using the Schwarz inequality that

$$
\left|x_{n k}(\lambda)\right| \leq\|S\| \leq 2\|A\|=2 \sup _{k} \frac{1}{2} \sqrt{\frac{(-k)(2 \lambda+k-1)}{(k+\lambda)(k+\lambda-1)}} \leq \sqrt{\frac{-2 \lambda n}{(n+\lambda)(\lambda+n-1)}} .
$$

Relation (2.6) follows immediately from (2.5).

Proof of Theorem 2.6. The Jacobi polynomials $P_{n}^{(a, \beta)}(x)$ satisfy the recurrence relation (3.1) for

$$
\begin{gathered}
\tilde{\alpha}_{n}=\frac{2(n+1)(n+a+\beta+1)}{(2 n+a+\beta+1)(2 n+a+\beta+2)}, \\
\tilde{\beta}_{n}=\frac{\beta^{2}-a^{2}}{(2 n+a+\beta+2)(2 n+a+\beta)}, \\
\tilde{c}_{n}=\frac{2(n+a)(n+\beta)}{(2 n+a+\beta+1)(2 n+a+\beta)} .
\end{gathered}
$$

However, by setting $P_{n}^{(a, \beta)}(x)=U_{n} Q_{n}^{(a, \beta)}(x)$, where $U_{-1}=0, U_{0}=1$, and

$$
U_{n+1}=\sqrt{\frac{(n+a+1)(n+\beta+1)(2 n+a+\beta+1)}{(n+1)(n+a+\beta+1)(2 n+a+\beta+3)}} U_{n}, \quad \text { for } n \geq 1
$$

one obtains as in [23] a relation for $Q_{n}^{(a, \beta)}(x)$ of the form (3.1) but now with

$$
\begin{gathered}
\tilde{\alpha}_{n}=\sqrt{\frac{4(n+1)(n+a+1)(n+\beta+1)(n+a+\beta+1)}{(2 n+a+\beta+2)^{2}(2 n+a+\beta+1)(2 n+a+\beta+3)}}=a_{n}, \\
\tilde{\beta}_{n}=\frac{\beta^{2}-a^{2}}{(2 n+a+\beta+2)(2 n+a+\beta)}=\beta_{n}, \quad \tilde{c}_{n}=a_{n-1} .
\end{gathered}
$$

For $a<-n, \beta<-n, a+\beta<-2(n+1)$ the sequence $\alpha_{n}$ is purely imaginary and can be rewritten as

$$
\alpha_{n}=i \sqrt{-\frac{4(n+1)(n+a+1)(n+\beta+1)(n+a+\beta+1)}{(2 n+a+\beta+2)^{2}(2 n+a+\beta+1)(2 n+a+\beta+3)}} .
$$


It is obvious from what already mentioned that the zeros $x_{n k}(a, \beta)$ of $P_{n}^{(a, \beta)}(x)$ are the same with the eigenvalues of the operator $T=A V^{*}+V A+B$, where

$$
\begin{gathered}
A E_{k}=i \sqrt{-\frac{4(k+1)(k+a+1)(k+\beta+1)(k+a+\beta+1)}{(2 k+a+\beta+2)^{2}(2 k+a+\beta+1)(2 k+a+\beta+3)}} E_{k}, \quad k=0, \ldots, n-1, \\
B E_{k}=\frac{\left(\beta^{2}-a^{2}\right)}{(2 k+a+\beta+2)(2 k+a+\beta)} E_{k}, \quad k=0, \ldots, n-1 .
\end{gathered}
$$

Due to (3.9), it is obvious that

$$
\operatorname{Re}\left[x_{n k}(a)\right]=\left(B f_{k}, f_{k}\right), \quad \operatorname{Im}\left[x_{n k}(a)\right]=\left(S f_{k}, f_{k}\right) .
$$

Since

$$
\begin{gathered}
\left(B f_{k}, f_{k}\right)=\sum_{l=0}^{n-1} \beta_{l}\left|\left(f_{k}, E_{l}\right)\right|^{2}, \\
\frac{\beta^{2}-a^{2}}{(2 n+a+\beta)(2 n+a+\beta-2)} \leq \beta_{l} \leq \frac{\beta-a}{a+\beta+2}, \quad \text { for } \beta>a, \\
\frac{\beta-a}{a+\beta+2} \leq \beta_{l} \leq \frac{\beta^{2}-a^{2}}{(2 n+a+\beta)(2 n+a+\beta-2)}, \quad \text { for } \beta<a,
\end{gathered}
$$

relation (2.7) follows as in the proof of Theorem 2.1.

Regarding the imaginary part of $x_{n k}(a, \beta)$, it follows using the Schwarz inequality that

$$
\begin{aligned}
& \left|\operatorname{Im}\left[x_{n k}(a, \beta)\right]\right| \leq\|S\| \leq 2\|A\| \\
& \quad \Longrightarrow\left|\operatorname{Im}\left[x_{n k}(a, \beta)\right]\right| \leq 2 \sup _{k} \sqrt{-\frac{4(k+1)(k+a+1)(k+\beta+1)(k+a+\beta+1)}{(2 k+a+\beta+2)^{2}(2 k+a+\beta+1)(2 k+a+\beta+3)}} \\
& \Longrightarrow\left|\operatorname{Im}\left[x_{n k}(a, \beta)\right]\right| \leq 2 \sqrt{\frac{-4(a+1)(\beta+1)(a+\beta+1) n}{\left[(2 n+a+\beta)^{2}-1\right](2 n+a+\beta)^{2}}} .
\end{aligned}
$$

Relation (2.9) follows immediately from relations (2.7) and (2.8). Relation (2.10) follows as in the proof of Theorem 2.1.

Proof of Theorem 2.10. The Al-Salam Carlitz II orthonormal polynomials $P_{n}^{(a)}(x ; q)$ satisfy the recurrence relation (3.1) for

$$
\tilde{\alpha}_{n}=\sqrt{\frac{a\left(1-q^{n+1}\right)}{q^{2 n+1}}}, \quad \tilde{\beta}_{n}=\frac{a+1}{q^{n}}, \quad \tilde{c}_{n}=\tilde{a}_{n-1},
$$


where $0<q<1$. For $a<0$, the sequence $\tilde{\alpha}_{n}$ is purely imaginary and can be rewritten as $\tilde{\alpha}_{n}=$ $i \sqrt{\left((-a)\left(1-q^{n+1}\right)\right) / q^{2 n+1}}$. It is obvious from what already mentioned that the zeros $x_{n k}(a ; q)$ of $P_{n}(x ; q)$ are the same with the eigenvalues of the operator $T=A V^{*}+V A+B$, where

$$
A E_{k}=i \sqrt{\frac{(-a)\left(1-q^{k+1}\right)}{q^{2 k+1}}} E_{k}, \quad B E_{k}=\frac{a+1}{q^{k}} E_{k}, \quad k=0, \ldots, n-1 .
$$

Due to (3.9), it is obvious that

$$
\operatorname{Re}\left[x_{n k}(a ; q)\right]=\left(B f_{k}, f_{k}\right), \quad \operatorname{Im}\left[x_{n k}(a ; q)\right]=\left(S f_{k}, f_{k}\right)
$$

Since

$$
\begin{gathered}
\left(B f_{k}, f_{k}\right)=\sum_{l=0}^{n-1} \beta_{l}\left|\left(f_{k}, e_{l}\right)\right|^{2}, \\
\frac{a+1}{q^{n-1}} \leq \beta_{l} \leq a+1, \quad \text { for } a \leq-1, \\
a+1 \leq \beta_{l} \leq \frac{a+1}{q^{n-1}}, \quad \text { for }-1<a<0,
\end{gathered}
$$

relation (2.12) follows as in the proof of Theorem 2.1.

Regarding the imaginary part of $x_{n k}(a ; q)$, it follows using the Schwarz inequality that

$$
\left|\operatorname{Im}\left[x_{n k}(a ; q)\right]\right| \leq\|S\| \leq 2\|A\|=2 \sup _{k} \sqrt{\frac{(-a)\left(1-q^{k+1}\right)}{q^{2 k+1}}} \leq 2 \sqrt{\frac{(-a)\left(1-q^{n}\right)}{q^{2 n-1}}} .
$$

Relation (2.14) follows as in the proof of Theorem 2.1.

Proof of Theorem 2.12. The $q$-Laguerre orthonormal polynomials $L_{n}^{(a)}(x ; q)$ satisfy the recurrence relation (3.1) for

$$
\tilde{\alpha}_{n}=\sqrt{\frac{\left(1-q^{n+1}\right)\left(1-q^{n+a+1}\right)}{q^{4 n+2 a+3}}}, \quad \tilde{\beta}_{n}=\frac{1-q^{n+1}+q-q^{n+a+1}}{q^{2 n+a+1}}, \quad \tilde{c}_{n}=\tilde{a}_{n-1},
$$

where $0<q<1$. For $a<-n$, the sequence $\tilde{\alpha}_{n}$ is purely imaginary and can be rewritten as $\tilde{\alpha}_{n}=i \sqrt{\left(\left(1-q^{n+1}\right)\left(q^{n+a+1}-1\right)\right) / q^{4 n+2 a+3}}$. It is obvious from what already mentioned that the 
zeros $x_{n k}(a ; q)$ of $L_{n}^{(a)}(x ; q)$ are the same with the eigenvalues of the operator $T=A V^{*}+V A+B$, where

$$
\begin{gathered}
A E_{k}=i \sqrt{\frac{\left(1-q^{k+1}\right)\left(q^{k+a+1}-1\right)}{q^{4 k+2 a+3}}} E_{k} \\
B E_{k}=\frac{1-q^{k+1}+q-q^{k+a+1}}{q^{2 k+a+1}} E_{k}, \quad k=0, \ldots, n-1 .
\end{gathered}
$$

Due to (3.9), it is obvious that

$$
\operatorname{Re}\left[x_{n k}(a ; q)\right]=\left(B f_{k}, f_{k}\right), \quad \operatorname{Im}\left[x_{n k}(a ; q)\right]=\left(S f_{k}, f_{k}\right)
$$

Since

$$
\begin{gathered}
\left(B f_{k}, f_{k}\right)=\sum_{l=0}^{n-1} \beta_{l}\left|\left(f_{k}, e_{l}\right)\right|^{2}, \\
\frac{1-q^{a+1}}{q^{2 n+a-1}} \leq \beta_{l} \leq \frac{1+q}{q^{2 n+a-1}}-\frac{q^{n+a}+q^{n}}{q^{a+1}},
\end{gathered}
$$

relation (2.15) follows as in the proof of Theorem 2.1.

Regarding the imaginary part of $x_{n k}(a ; q)$, it follows using the Schwarz inequality that

$$
\begin{aligned}
\left|\operatorname{Im}\left[x_{n k}(a ; q)\right]\right| & \leq\|S\| \leq 2\|A\|=2 \sup _{k} \sqrt{\frac{\left(1-q^{k+1}\right)\left(q^{k+a+1}-1\right)}{q^{4 k+2 a+3}}} \\
& \Longrightarrow\left|\operatorname{Im}\left[x_{n k}(a ; q)\right]\right| \leq 2 \sqrt{\frac{\left(1-q^{n}\right)\left(q^{a+1}-1\right)}{q^{4 n+2 a-1}}} .
\end{aligned}
$$

\section{References}

[1] W. Gautschi, "Orthogonal polynomials: applications and computation," in Acta Numerica, vol. 5 of Acta Numer, pp. 45-119, Cambridge University Press, Cambridge,UK, 1996.

[2] R. Álvarez-Nodarse, Polinomios Hipergeométricos Clásicos y q-Polinomios, vol. 26 of Monografías del Seminario Matemático "Garcia de Galdeano", Universidad de Zaragoza Seminario Matematico "Garcia de Galdeano", Zaragoza, Spain, 2003.

[3] T. S. Chihara, An Introduction to Orthogonal Polynomials, vol. 13 of Mathematics and Its Applications, Gordon and Breach Science Publishers, New York, NY, USA, 1978.

[4] M. E. H. Ismail, Classical and Quantum Orthogonal Polynomials in One Variable, vol. 98 of Encyclopedia of Mathematics and Its Applications, Cambridge University Press, Cambridge, UK, 2005.

[5] R. Koekoek and R. F. Swarttouw, "The Askey-scheme of hypergeometric orthogonal polynomials and its $q$-analogue," Tech. Rep. 98-17, Delft University of Technology, Faculty of Information Technology and Systems, Department of Technical Mathematics and Informatics, Delft, The Netherlands, 1998.

[6] G. Szego, Orthogonal Polynomials, vol. 23, American Mathematical Society, Providence, RI, USA, 4th edition, 1975, American Mathematical Society. 
[7] C. Berg and A. Ruffing, "Generalized q-Hermite polynomials," Communications in Mathematical Physics, vol. 223, no. 1, pp. 29-46, 2001.

[8] J. S. Christiansen, "The moment problem associated with the $q$-Laguerre polynomials," Constructive Approximation, vol. 19, no. 1, pp. 1-22, 2003.

[9] J. S. Christiansen and E. Koelink, "Self-adjoint difference operators and symmetric Al-Salam-Chihara polynomials," Constructive Approximation, vol. 28, no. 2, pp. 199-218, 2008.

[10] E. K. Ifantis and P. D. Siafarikas, "Differential inequalities for the largest zero of Laguerre and Ultraspherical polynomials," in Proceedings of the Actas del VI Symposium on Polinomios Orthogonales y Applicationes, vol. 49, pp. 187-197, Gijon, Spain.

[11] E. K. Ifantis and P. D. Siafarikas, "On the zeros of a class of polynomials including the generalized Bessel polynomials," Journal of Computational and Applied Mathematics, vol. 49, no. 1-3, pp. 103-109, 1993.

[12] E. N. Petropoulou, P. D. Siafarikas, and I. D. Stabolas, "Convexity results for the largest zero and functions involving the largest zero of $q$-associated polynomials," Integral Transforms and Special Functions, vol. 16, no. 2, pp. 171-178, 2005.

[13] P. D. Siafarikas, I. D. Stabolas, and L. Velazquez, "Differential inequalities of functions involving the lowest zero of some associated orthogonal q-polynomials," Integral Transforms and Special Functions, vol. 16, no. 4, pp. 337-376, 2005.

[14] P. J. Forrester and J. B. Rogers, "Electrostatics and the zeros of the classical polynomials," SIAM Journal on Mathematical Analysis, vol. 17, no. 2, pp. 461-468, 1986.

[15] A. F. Nikiforov, S. K. Suslov, and V. B. Uvarov, Classical Orthogonal Polynomials of a Discrete Variable, Springer Series in Computational Physics, Springer, Berlin, Germany, 1991, Translated from the Russian.

[16] A. F. Nikiforov and V. B. Uvarov, Special Functions of Mathematical Physics. A Unified Introduction with Applications, Birkhauser, Basel, Switzerland, 1988, Translated from the Russian and with a preface by R. P. Boas. With a foreword by A. A. Samarskii.

[17] M. V. DeFazio, On the zeros of some quasi-definite orthogonal polynomials, Ph.D. thesis, Ontario, Canada, 2001.

[18] M. V. DeFazio, D. P. Gupta, and M. E. Muldoon, "Limit relations for the complex zeros of Laguerre and $q$-Laguerre polynomials," Journal of Mathematical Analysis and Applications, vol. 334, no. 2, pp. 977-982, 2007.

[19] K. Driver and P. Duren, "Zeros of the hypergeometric polynomials $F(-n, b ; 2 b ; z)$," Indagationes Mathematicae, vol. 11, no. 1, pp. 43-51, 2000.

[20] K. Driver and P. Duren, "Zeros of ultraspherical polynomials and the Hilbert-Klein formulas," Journal of Computational and Applied Mathematics, vol. 135, no. 2, pp. 293-301, 2001.

[21] E. K. Ifantis and P. D. Siafarikas, "Differential inequalities and monotonicity properties of the zeros of associated Laguerre and Hermite polynomials," Annals of Numerical Mathematics, vol. 2, no. 1-4, pp. 79-91, 1995.

[22] C. G. Kokologiannaki and P. D. Siafarikas, "Convexity of the largest zero of the ultraspherical polynomials," Integral Transforms and Special Functions, vol. 4, no. 3, pp. 275-278, 1996.

[23] E. K. Ifantis, C. G. Kokologiannaki, and P. D. Siafarikas, "Newton sum rules and monotonicity properties of the zeros of scaled co-recursive associated polynomials," Methods and Applications of Analysis, vol. 3, no. 4, pp. 486-497, 1996. 


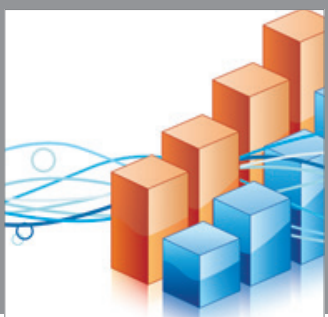

Advances in

Operations Research

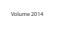

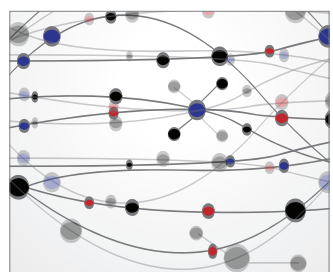

\section{The Scientific} World Journal
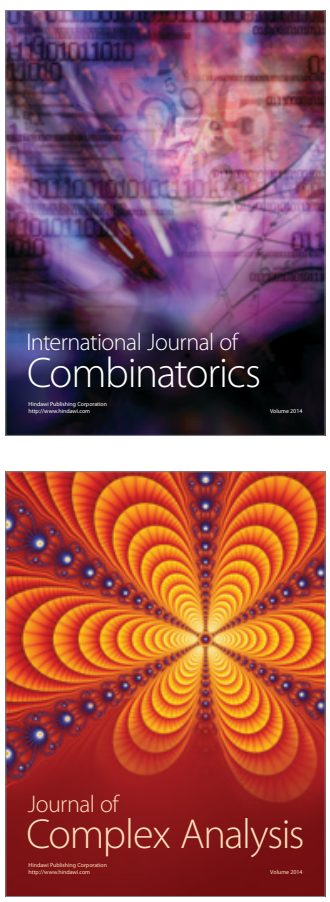

International Journal of

Mathematics and

Mathematical

Sciences
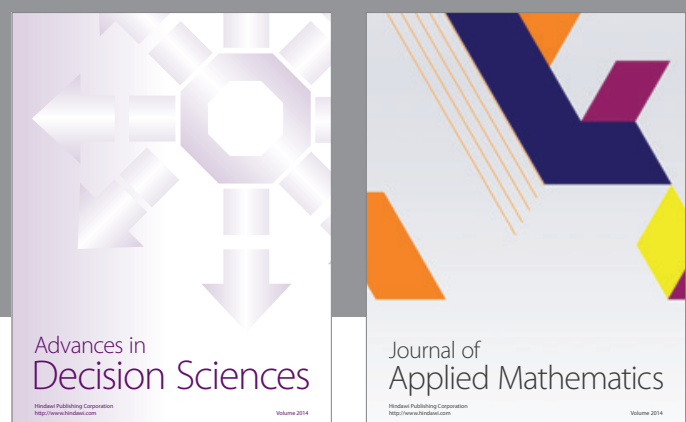

Journal of

Applied Mathematics
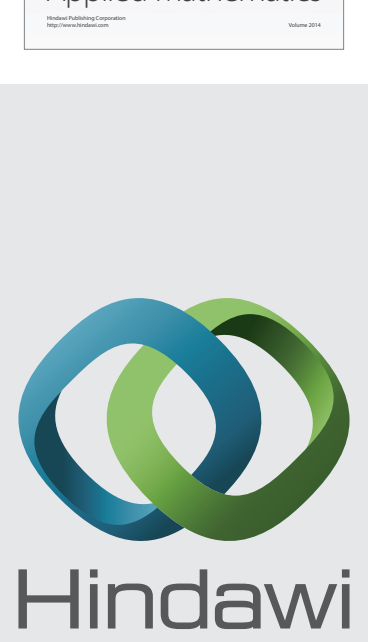

Submit your manuscripts at http://www.hindawi.com
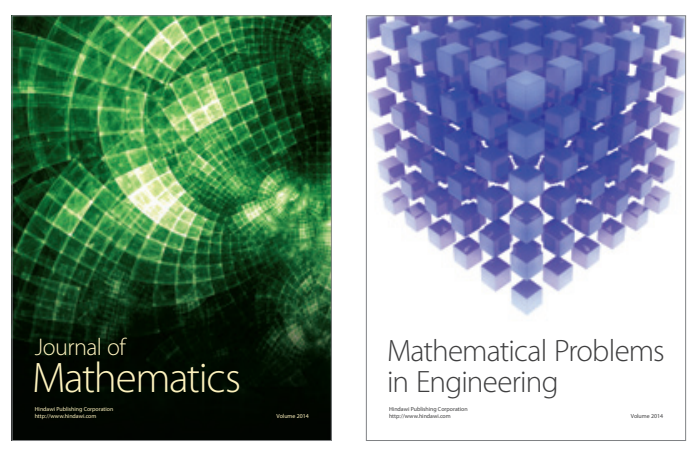

Mathematical Problems in Engineering
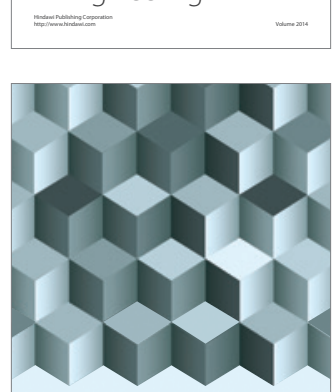

Journal of

Function Spaces
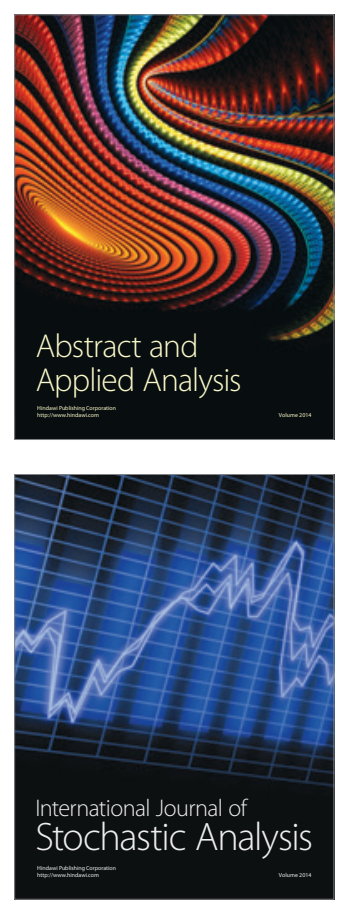

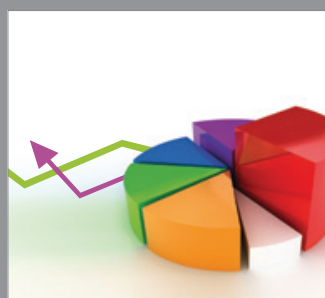

ournal of

Probability and Statistics

Promensencen
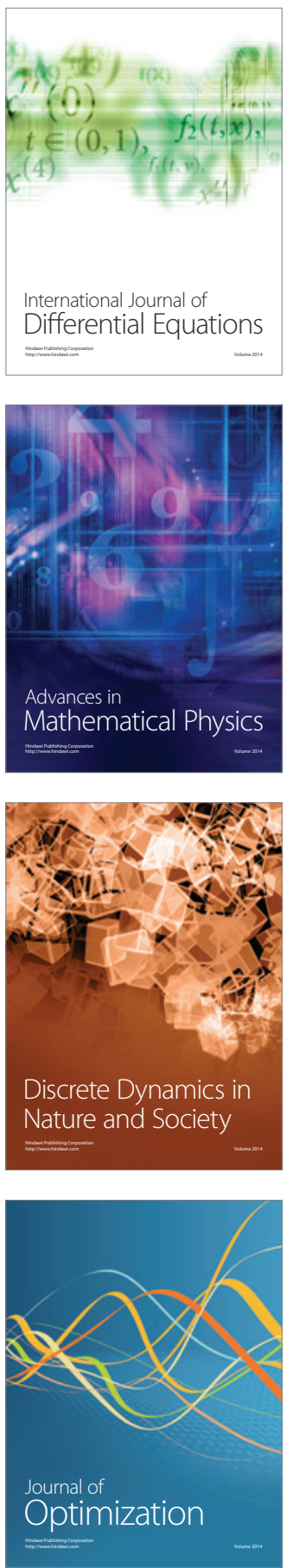